\title{
Alternative Konzepte zur Integration und zum Auslesen von Drucksensoren
}

\author{
Remigius R. Poloczek ${ }^{1}$, Christopher Kontis ${ }^{1}$, Klaus T. Kallis ${ }^{1}$, Horst L. Fiedler ${ }^{1}$ \\ ${ }^{1} T U$ Dortmund, Lehrstuhl für Intelligente Mikrosysteme, Emil-Figge-Str. 68, 44227 Dortmund
}

\section{Zusammenfassung}

Zur Realisierung von Drucksensoren ist die Wandlung der druckbedingten mechanischen Verformung einer Membran in ein elektrisches Signal unerlässlich. Im Rahmen des Artikels werden hierfür innovative Möglichkeiten zur monolithischen Integration in Silicon-on-Insulator, kurz SOI-Substrat aufgezeigt. Diese ermöglichen neben der Einsparung von Herstellungskosten die Senkung des Energieverbrauches. Die Wandlung der mechanischen Spannung in ein elektrisches Signal erfolgt mittels eines Transistors und nicht wie in konventionellen Lösungen durch beispielsweise die Verstimmung einer Wheatstone'schen-Brückenschaltung oder eine Kapazitätsänderung. Zur weiteren Senkung der Kosten findet ebenfalls die Betrachtung von konventionellem Silizium Substrat statt, wobei der Fokus auf der Optimierung einer im Niedertemperaturverfahren abgeschiedenen amorphen Siliziumschicht liegt.

Keywords: MEMS, Druckmessung, monolithische Integration, SOI-Substrat, amorphes Silizium

\section{Einleitung}

Eine Schlüsselkomponente in Mikro-ElektroMechanischen-Systemen (MEMS) stellt oftmals der Drucksensor dar. Den Primärmarkt bildet hierbei die Automobilindustrie, wobei der Einsatz nicht nur auf die seit November 2014 gesetzlich vorgeschriebene Überwachung des Reifenfülldruckes beschränkt ist.

Eine hohe Anzahl an Anwendungen findet sich zudem im Bereich der Bio- sowie der Medizintechnik [1]. Infolge sinkender Abmessungen und Herstellungskosten ermöglichen Drucksensoren neue Wege in der Überwachung der Körperfunktionen, beispielsweise durch eine unmittelbare Messung der Körperflüssigkeiten in der Blutbahn, der Blase oder dem Darm. Neben der zunehmenden Miniaturisierung erfolgt die Erweiterung zu komplexen Datenerfassungssystemen. Diese verfügen über eine Energieversorgung, Datenaufbereitung und Übermittlung und ermöglichen somit eine Implantation. Hierdurch wird die Messung des Augeninnendruckes ohne große Einschränkung des Patienten ermöglicht und eine Beeinträchtigung durch die Behandlung von Krankheiten, beispielsweise der Augenkrankheit Glaukom (,Grüner Star") erheblich gemindert. [2] Der Einsatz solcher Druckerfassungssysteme eröffnet daher neue Möglichkeiten der Diagnostik. Druckmessungen des Augenliddruckes durch das Aufbringen des Systems auf eine Kontaktlinse werden beispielsweise möglich. [3]
Die fortschreitende Skalierung bedingt ebenfalls eine Senkung des Sensorgewichtes, was bei gleichzeitiger Erhöhung der Empfindlichkeit durch freitragende Strukturen den Druck auf Insektenflügeln erfassbar macht. Dies wiederum trägt erheblich zum Verständnis der Natur bei und liefert neue Erkenntnisse und Möglichkeiten für technische Realisierungen. [4]

\section{Konzepte zur Integration}

Die Herstellung moderner Sensoren erfolgt häufig als System-in-Package (SiP) Lösung. Hierbei werden die mechanische Komponente und die elektrische Schaltung des Sensors unabhängig voneinander prozessiert und erst durch Bonddrähte im Gehäuse zu einem vollständigen Sensorsystem verbunden. Dieses Verfahren ermöglicht das Testen der Einzelkomponenten und senkt somit den Ausschuss, da lediglich funktionsfähige Komponenten zu einem Gesamtsystem verbunden werden. Die Verbindung durch entsprechende Bonddrähte hat jedoch im Bereich der Signalqualität, insbesondere beim Rauschen, Nachteile gegenüber einer Kontaktierung auf Waferebene. [5] Diese wird bei der gegenüber dem SiPVerfahren selten eingesetzten System-on-Chip (SoC) Technologie realisiert. Zur Herstellung solcher Sensorsysteme wird die etablierte und für die Massenproduktion kostengünstige Complementary - Metal - Oxide - Semiconductor kurz CMOS-Technologie verwendet. Bei der 
Realisierung von Sensorsystemen in der SoCTechnologie werden mechanische und elektrische Komponenten auf dem gleichen Substrat prozessiert. Neben der erwähnten Vorteile mit Blick auf die Verdrahtung führt dies zu einem geringeren Gesamtflächenbedarf. [6] Dabei kann die Integration der zur Herstellung mikromechanischer Strukturen erforderlichen Schritte in die konventionelle Prozessabfolge der Herstellung von elektrischen Bauelementen prinzipiell auf drei Arten geschehen:

- Vor dem eigentlichen Prozessablauf

- nach der Metallisierung

- in den Prozessablauf eingebettet.

Vorausgesetzt, dass die Fertigung der elektrischen Schaltung in einer unternehmensfremden Produktionslinie erfolgt, müssen die mechanischen Komponenten des Sensors enthaltenden und somit vorprozessierten Substrate von dem entsprechenden Linienbetreiber akzeptiert werden. Da dies selten der Fall ist, kommt dieses Verfahren nicht häufig zum Einsatz, obwohl sich hierbei die geringsten Einschränkungen bezüglich Prozesstemperaturen bei der Herstellung der mechanischen Komponenten ergeben. Eine weitere Möglichkeit der Integration der mechanischen Strukturfertigung in den CMOS-Ablauf bietet sich vor dem Vereinzeln und dem Einbau des Sensorsystems in ein Gehäuse an. Vorteile bietet hierbei die Tatsache, dass bei diesem Verfahren der geringste Eingriff in standardisierte Abläufe zur Schaltungsherstellung erfolgt und die Fertigung der elektrischen Komponente in einer beliebigen standardisierten CMOS-Prozesslinie realisiert werden kann. Nachteilig ist bei diesem Verfahren jedoch das zur Verfügung stehende Temperaturbudget, da es nach der Metallisierung der Schaltung ansetzt. Daher wird die Temperatur zur Herstellung der mechanischen Komponente auf rund 350 bis $400^{\circ} \mathrm{C}$ limitiert, was wiederum die Werkstoffauswahl und Schichtqualität erheblich eingegrenzt. Alternativ kann die Herstellung der Strukturen unmittelbar in den Ablauf der Schaltungsherstellung integriert werden. Dies hat zur Folge, dass die Temperaturbeschränkung lediglich von vorangegangenen Schritten und dem aktuellen Prozess abhängig ist. Des Weiteren führt die Zusammenführung der erforderlichen Prozessschritte und lithographischen Masken zu einer Kosteneinsparung. Zu beachten ist bei diesem Verfahrensablauf jedoch, dass die zur gleichzeitigen Herstellung der mechanischen Sensorkomponente erforderlichen Abläufe die gleichzeitig prozessierten elektrischen Bauteile beispielsweise durch zusätzliche Schichtabscheidung nicht unerheblich beeinflussen.
Unter Abwägung aller Vor- und Nachteile bietet daher die Fertigung der mechanischen Komponente nach der Metallisierung das höchste Potential. [5],[6] Diese erfordert jedoch weitere Forschung und Entwicklung im Bereich der Abscheidung von qualitativ hochwertigen Schichten bei geringeren Temperaturen (kleiner $\left.400^{\circ} \mathrm{C}\right)$. [7]

\section{Konzepte zur Signalwandlung}

Neben dem Herstellungsverfahren ist beim Entwurf moderner Sensorsysteme die Wahl der Wandlung der mechanischen Messgröße in ein elektrisches Signal entscheidend. Innovative Möglichkeiten zur monolithischen Integration solcher Wandler ermöglichen neben der Einsparung von Herstellungskosten die Senkung des Energieverbrauches. Konventionellen Lösungen durch Signalwandlung nutzen beispielsweise die Verstimmung einer Wheatstone'schen-Brückenschaltung [8] oder die Änderung einer Kapazität [9]. Aufbauend auf den Untersuchungen von Zhang et al. [10] erfolgt die Wandlung der druckbedingten Deformation in ein elektrisches Signal mittels eines Transistors. Während in der erwähnten Publikation lediglich zwei Widerstände einer Brückenschaltung durch Transistoren ersetzt werden, beruht die Wandlung im vorliegenden Grundkonzept auf der Nutzung eines Transistors. Mechanische Spannungen im Kanalbereich eines Feldeffekttransistors (FET) führen zu einer Änderung des Drainstroms. Druckspannungen führen bei positiven Ladungsträgern zu einem entsprechenden Anstieg des Drainstroms [11]. Bei negativen Ladungsträgern stellt sich dieser Effekt entsprechend bei einer Zugbelastung des Kanalmaterials ein [12]. Darauf aufbauend erfolgt die Positionierung des Kanalbereiches eines FETs auf der Membran. Die mechanische Verspannung infolge einer druckabhängigen Durchbiegung wird im Transferkennlinienfeld somit durch eine Änderung des Drainstromes beobachtet. Die Verwendung eines Feldeffekttransistors mit nanoskaligen Gateabmessungen im Unterschwellenspannungsbereich bietet neben einer weiteren Möglichkeit der Sensitivitätssteigerung durch den exponentiellen Zusammenhang zwischen Messgröße und Signal den Vorteil eines geringeren Energieverbrauches des Sensorsystems im Betrieb [13].

Hierfür stehen zwei Konzepte im Fokus. Zum einen erfolgt die Prozessierung der Auswerteschaltung und somit der elektrischen Sensorkomponente auf einer zuvor hergestellten Membran. Als Grundlage bietet sich hierbei die Verwendung von Silicon-on-Insulator (SOI) Substrat an. Dies ermöglicht die Herstellung einer monokristallinen Membran, deren Ablauf eine vollständige Kompatibilität zur Schaltkreisherstellung sicherstellt [14]. 
Zum anderen kann die mechanische Sensorkomponente über einer zuvor in konventioneller Art auf Standardsubstrat hergestellten elektrischen Schaltung realisiert werden. Als Membran dient hierbei eine Schicht aus amorphen Silizium, welche in Niedertemperaturverfahren hergestellt wird. Diese eignet sich aufgrund der Materialeigenschaften nur bedingt zur Implementierung von Transistoren. Aufgrund der Verwendung dieser im Unterschwellenspannungsbereich sind die einhergehenden Eigenschaften, wie beispielsweise Leckströme, unbedenklich und verstärken bisweilen die Wandlung der Deformation in ein elektrisches Signal. Ein einzelner Metal-OxideSemiconductor (MOS) FET wird hierfür mit dem Kanalgebiet an der Stelle der maximalen Spannung, also bei einer rechteckigen Membran in der Mitte einer Kante, platziert. Abbildung 1 zeigt schematisch einen solchen Transistor, bei dem das Kanalgebiet Abmessungen im sub $50 \mathrm{~nm}$ Bereich aufweist, wobei lediglich der Top-Layer ohne Kavität und restlichem Substrat dargestellt ist.

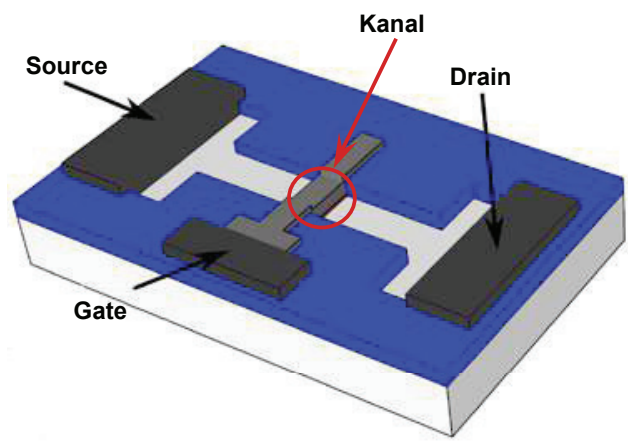

Abb.. 1: Schematische Skizze eines MOSFETs mit nanoskaligen Gateabmessungen zur Wandlung der mechanischen Spannung in ein elektrisches Signal.

Der Transistor wird nach der noch im Folgenden beschriebenen Erzeugung der Membran realisiert. Auf dem $p$-vordotierten Toplayer wird zunächst ein Oxid im Plasma-EnhancedChemical-Vapour-Deposition (PECVD) Verfahren als Zwischenoxid abgeschieden. Dem schließt sich das Strukturieren der Drain- und Sourcegebiete sowie des Kanalbereiches an. Zur Einhaltung der gewünschten Kanalabmessungen wird auf die Elektronenstrahllithographie zurückgegriffen, wobei zur besseren Kontaktierung von Drain und Source die Öffnung im Oxid vergrößert wird. Der so partiell freigelegte Toplayer wird im Folgenden thermisch oxidiert, wodurch das Gatedielektrikum realisiert wird. Dem schließt sich die Abscheidung des Gatematerials an. Hierfür wird amorphes Silizium im PECVD-Prozess abgeschieden und entsprechend strukturiert. Nach der Abschei- dung eines Streuoxides erfolgt die Aktivgebietsimplantation, wobei die Gateelektrode ebenfalls eine Anreicherung an Donatoren erhält, was zu einer Verbesserung der Leitfähigkeit führt. Nach dem Entfernen des Oxides erfolgt die Kontaktierung der Elektroden. Aufgrund der notwendigen thermischen Oxidation und einer Aktivierungstemperung nach der Implantation eignet sich dieses Konzept weniger zur Realisierung auf einer zuvor gefertigten Schaltung.

Die Grundlage zur Möglichkeit der monolithischen Integration des Sensors und dem Nachweis der Funktionalität der vorgestellten Konzepte veranschaulicht der in Abbildung 2 schematisch gezeigte und auf der SOITechnologie basierende Prozessablauf zur Herstellung der mechanischen Grundstruktur.

a)

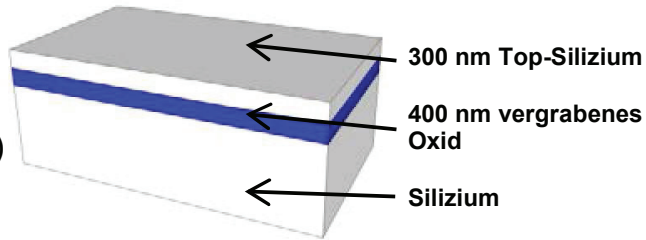

c)
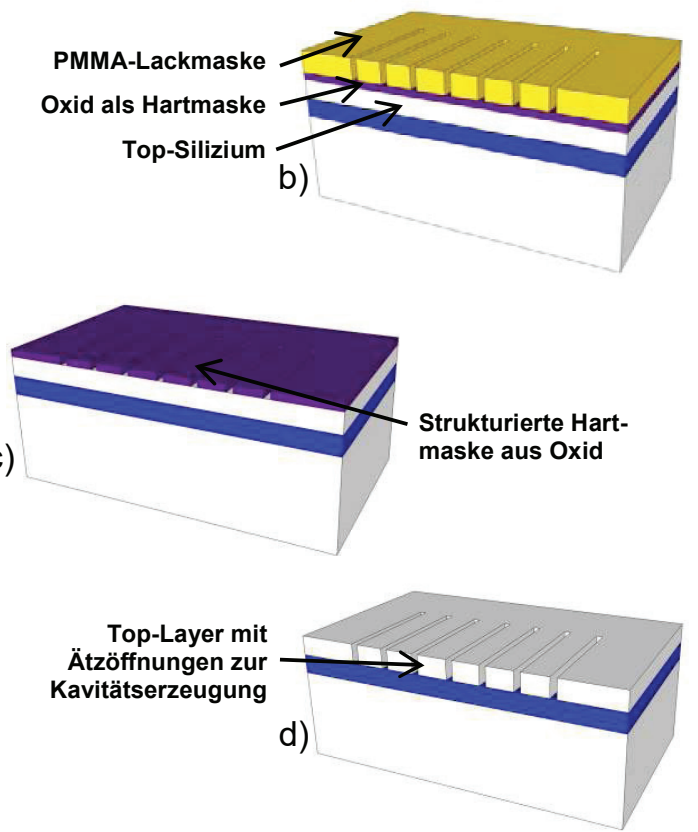

e)

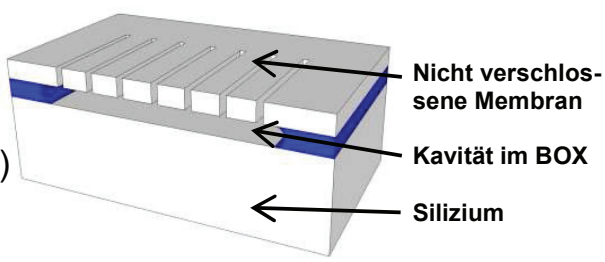

Abb.. 2: Schematische Darstellung des Prozessablaufes zur Herstellung der mechanischen Komponente des Sensors.

Das Ausgangssubstrat ist SOI mit einer $300 \mathrm{~nm}$ dicken Silizium-Nutzschicht (TopSilizium) und einer vergrabenen Oxidschicht 
(BOX) von $400 \mathrm{~nm}$ Dicke (Abb. 2, a). Die für die trockenchemische Strukturierung erforderliche Maskierung wird zur Erzielung von Öffnungen mit sub $100 \mathrm{~nm}$ Abmessungen mittels der Elektronenstrahllithographie realisiert. Der eingesetzte Lack, bestehend aus Polymethylmethacrylat (PMMA) weist jedoch eine zu geringe Selektivität beim Ätzen des Siliziums auf. Daher ist eine Hartmaske aus Siliziumdioxid erforderlich, welche in einem ersten Schritt strukturiert wird (Abb. 2, b und c). Dem schließt sich die eigentliche anisotrope Ätzung des Top-Siliziums an (Abb. 2, d), gefolgt vom isotropen Entfernen des vergrabenen Oxides zur Herstellung der Kavität (Abb. 2, e).

Die resultierende, aufgrund der Anordnung der Ätzöffnungen quadratische Membran, weist eine Kantenlänge von $25 \mu \mathrm{m}$ auf. Hierdurch wird sichergestellt, dass ein Anhaften der Membran während des Trocknens („Sticking“) konstruktiv unterbunden wird.

Zur Analyse der Eignung eines Transistors zur Wandlung der mechanischen Verformung in ein elektrisches Signal wird auf einen aufwendig herzustellenden MOS-FET jedoch verzichtet und auf einen Pseudo-MOS-FET, kurz $\psi$ MOS-FET, zurückgegriffen, dessen Herstellungsaufwand mit der Realisierung einer Brückenschaltung vergleichbar ist. Der einfache Aufbau dieses Transistors führt dazu, dass dieser zur Charakterisierung von SOISubstraten verwendet werden kann. Abbildung 3 zeigt schematisch die Realisierung zweier Transistoren auf einem solchen Substrat

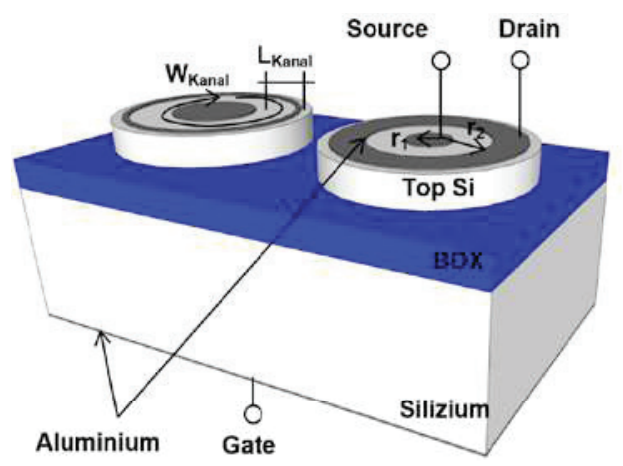

Abb.. 3: Schematische Darstellung zweier $\psi$ MOS-FETS mit unterschiedlichen Kanalabmessungen.

Durch Strukturierung des Top-Siliziums bis auf das vergrabene Oxid werden einzelne Transistoren auf dem Substrat voneinander isoliert. Die Rückseite des Trägersubstrates wird metallisiert und fungiert als gemeinsamer Gateanschluss. Drain und Source werden in Form von Metallringen auf der Oberfläche realisiert. Mangels einer Dotierung der Drainund Sourcebereiche stellt sich ein ambipolares Schaltverhalten ein. Dies bedeutet, dass sowohl positive als auch negative Gate- spannungen zu einem leitfäghigen Kanal führen. Die Abhängigkeit des Drainstroms von der Drain- und Gatespannung ist gemäß GI. (1) gegeben zu:

$I_{D}=f_{G} \cdot C_{O x} \cdot \mu_{n, p} \cdot \frac{\left(U_{G S}-U_{T h}\right) \cdot U_{D S}}{1+\theta \cdot\left(U_{G S}-U_{T h}\right)}$

wobei der Faktor $f_{G}$ den Quotienten aus Kanalweite sowie -länge angibt und für die in Abbildung 1 dargestellte Struktur nicht ohne weiteres ermittelt werden kann. Er ist gemäß GI. (2) zu:

$$
f_{G}=\frac{W_{\text {Kanal }}}{L_{\text {Kanal }}}=\frac{2 \cdot \pi}{\ln \left(\frac{r_{2}}{r_{1}}\right)}
$$

zusammengefasst. Die jeweilige Ladungsträgerbeweglichkeit entspricht dem Faktor $\mu_{n, p}$. Die Reduktion der eigentlichen Beweglichkeit durch den Bahnwiderstand zwischen Drain und Source fasst der Faktor $\theta$ zusammen. Die Schwellenspannung $U_{T h}$ in der Gleichung hat nur für den Fall eines invertierten Kanals Gültigkeit. Für den Fall der Akkumulation ist entsprechend die Flachbandspannung $U_{F B}$ einzusetzen. [15]

Zunächst erfolgt die Implementierung des $\psi$ MOS-Feldeffekttransistors auf der hergestellten mechanischen Struktur. Der so entstehende Drucksensor ist in Abbildung 4 schematisch dargestellt.

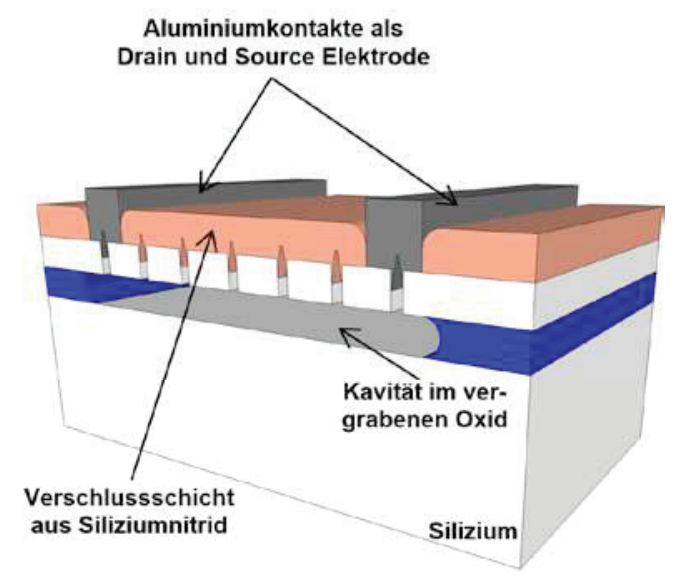

\section{Abb.. 4: Schematische Darstellung des Drucksensors mit einem $\psi$-MOS-FET als Wandlerelement.}

Dabei werden ebenfalls die einzelnen Drucksensoren durch eine Ätzung bis auf die vergrabene Oxidschicht voneinander isoliert. Die Realisierung des Drain- bzw. Sourcekontaktes wird nicht rund, sondern analog zur Geometrie der Membran rechteckig durch zwei Metallstreifen aus Aluminium ausgeführt. Das daraus 
resultierende Maskendesign ist Abbildung $5 \mathrm{zu}$ entnehmen.

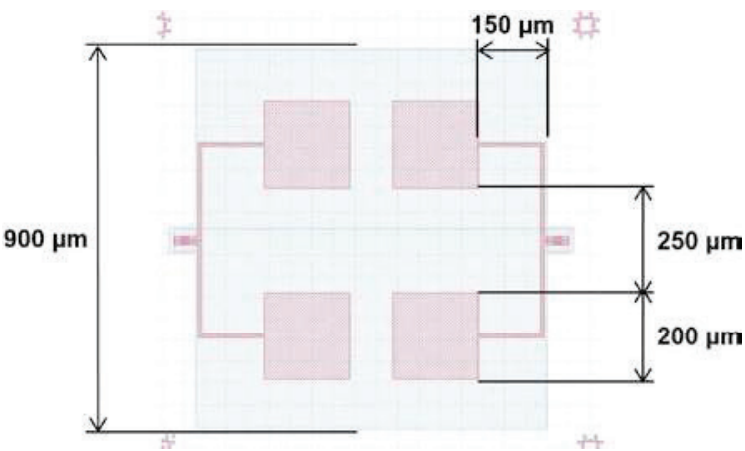

Abb.. 5: Maskenlayout mit Abmessungen zur Herstellung der beschriebenen Drucksensoren.

Damit die Strukturen beim Verbinden der Sensoren mit dem Gehäuse mittels eines Ultraschallbondverfahrens nicht beschädigt werden, sind relativ lange Zuleitungen zu den Kontaktflächen erforderlich. Der vorgestellte Transistor bildet die Grundlage der im Folgenden beschriebenen elektrischen Messungen.

\section{Ergebnisse}

Zunächst erfolgt die Betrachtung des $\psi$-MOSFETs als Element zur Wandlung der mechanischen Membranverspannung in ein elektrisches Auswertesignal. Die Messung erfolgt an einem gebondeten Sensor mit einer quadratischen Membranfläche einer Kantenlänge von $25 \mu \mathrm{m}$ und einer Kanallänge von $4 \mu \mathrm{m}$. Zur Untersuchung wird das Transferkennlinienfeld für negative Gate-Source-Spannungen ohne Druck sowie bei einem Überdruck von vier Bar gegenüber Atmosphäre aufgenommen und ist in Abbildung 6 dargestellt.

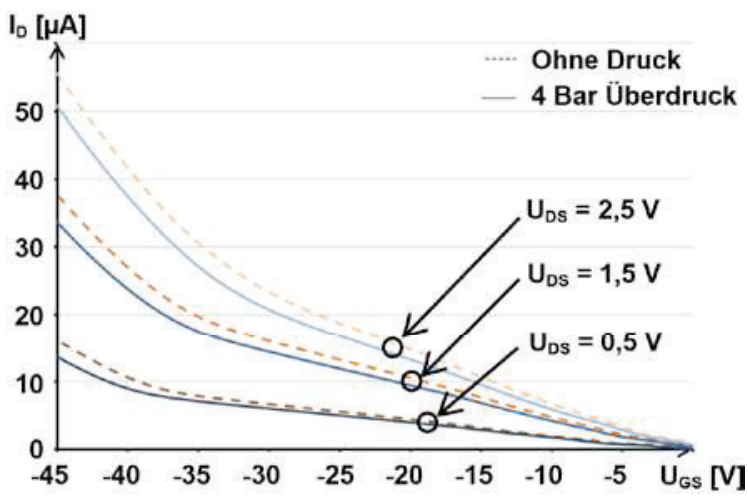

\section{Abb.. 6: Druckabhängige Änderung des Drainstroms dargestellt im Transfer- kennlinienfeld des Transistors.}

Der druckabhängige Verlauf des Drainstroms ist in erster Line durch eine veränderte Ladungsträgerbeweglichkeit zu erklären. Wie bereits erörtert, führt eine Druckspannung im Kanalgebiet bei positiven Ladungsträgern zu einer Erhöhung der Beweglichkeit und somit zu einem Anstieg des Stroms. Im vorliegenden Fall tritt aufgrund der druckbedingten Auslenkung der Membran eine Längung auf, welche Zugspannungen im entsprechenden Transistorbereich verursachen, was sich im Abfall des Drainstroms wiederspiegelt. Gleichzeitig ändert sich aber auch der Abstand zum Gate und somit die Dicke des Gatedielektrikums. Da der Drainstom gemäß GI. (1) proportional zur Gatekapazität ist, führt die Auslenkung zu einem Stromanstieg und verschlechtert somit die Sensitivität.

Wird der Auswertetransistor mit positiven Gate-Source-Spannungen betrieben, so überlagern sich die beschriebenen Effekte, da sich die Beweglichkeit der Elektronen beim Auftreten von Zugspannungen erhöht. In der Messung tritt jedoch ein durch Elektronen verursachter Strom nicht auf. Die Ursache hierfür liegt in der Vereinfachung des Prozessablaufes. So werden Drain und Source durch Aluminiumringe realisiert. Bildet sich nun im TopLayer infolge einer positiven Gate-SourceSpannung ein Kanal aus Elektronen aus, entsteht ein Übergang zwischen n-Halbleiter und Aluminium, was bevorzugt zu SchottkyKontakten führt. Durch unterschiedliche Austrittsarbeiten zwischen Metall und Halbleiter sowie unterschiedliche Elektronenkonzentration an der Grenzfläche bildet sich eine Potentialdifferenz, welche überwunden werden muss. Zudem ist Aluminium für den Halbleiterwerkstoff Silizium ein Akzeptor, wodurch bei der Metallisierung mittels Sputtern im Übergangsbereich eine dünne, jedoch stark p-dotierte Schicht entstehen kann, welche als Diode fungiert und somit den Nutzstrom weiter abschwächt. Durch die Metallisierung mittels einer Schichtenfolge aus Titannitrid, Titan und Aluminium kann dieser Effekt unterdrückt werden. [16]

Um den beschrieben Drucksensor nicht weiter auf kostenintensiven SOI-Substrat herstellen zu müssen, wird im weiteren Verlauf der Untersuchung amorphes Silizium als Funktionsschicht analysiert. Um diese über einer vorprozessierten Schaltung als Membran nutzen zu können, wird ausschließlich auf Niedertemperaturverfahren zurückgegriffen. Hierbei bietet das PECVD-Verfahren eine gute Möglichkeit zur Schichtabscheidung. Die abzuscheidende Schicht ist nicht nur hinsichtlich der elektrischen Eigenschaften zu optimieren, sondern muss mechanische Stabilität und eine gute Beständigkeit gegenüber Flusssäure aufweisen. Da für qualitativ hochwertige Schichten eine möglichst hohe Temperatur erforderlich ist, wird diese durch die im Substrat implementierte Schaltung begrenzt und beträgt $400^{\circ} \mathrm{C}$. Eine Optimierung der Schicht erfolgt durch die Variation des Prozessdruckes, des Reaktions- 
gasflusses von Silan, und der hochfrequenten Leistung. Tabelle 1 stellt die optimierten Prozessparameter dar.

Tab. 1: Prozessparameter zur Erzeugung der amorphen Siliziumschicht mittels PECVD

\begin{tabular}{|c|c|}
\hline Parameter & Wert \\
\hline Temperatur & $400^{\circ} \mathrm{C}$ \\
\hline Druck & $1000 \mathrm{mTorr}$ \\
\hline Gasfluss & $300 \mathrm{sccm}$ \\
\hline Leistung (HF) & $200 \mathrm{~W}$ \\
\hline Depositionszeit & 10 Minuten \\
\hline Evakuieren & 60 Minuten \\
\hline
\end{tabular}

Ein höherer Druck und ein größerer Gasfluss resultieren jeweils in einer porösen und somit nicht Flusssäure beständigen Schicht. Ein Prozessdruck im ausgewiesenen Bereich liefert das Optimum. Die Beständigkeit wird mit steigender Leistung verbessert, wobei diese anlagenseits auf 300 Watt limitiert ist. Da ein reproduzierbarer Prozessablauf bei Maximalleistung aufgrund von Plasmaschwankungen nicht realisiert werden kann, wird die Leistung auf den angegebenen Wert für folgende Untersuchungen limitiert. Für später folgende Vergleiche der Sensorkonzepte beträgt die Zieldicke der Schicht rund $250 \mathrm{~nm}$, wodurch sich eine entsprechende Abscheidezeit ergibt. Es stellt sich eine erwartete zeitlich lineare Schichtdickenzunahme ein. Um den Einbau von Fremdatomen zu vermeiden, wird eine entsprechend lange Evakuierungszeit vorgesehen, die eventuell vorhandene Moleküle aus dem Rezipienten entfernen soll. Abbildung 7 zeigt einen Querschnitt durch eine entsprechend gefertigte Schichtenfolge.

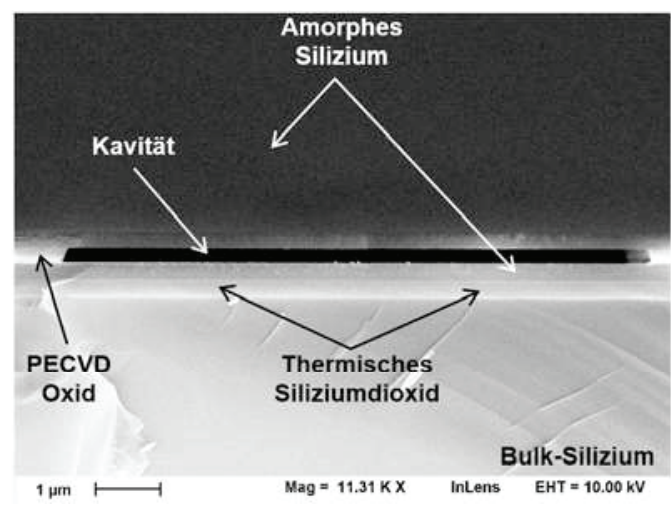

Abb.. 7: Rasterelektronenmikroskopaufnahme des Querschnittes einer Membran aus amorphen Silizium auf konventionellem Substrat.

Die thermische Siliziumdioxidschicht dient lediglich als Isolierung zum Substrat und ist bei der Herstellung eines Sensors auf einer im Bulksilizium integrierten Schaltung ebenfalls durch ein PECVD-Oxid zu ersetzen. Die darauf befindliche amorphe Siliziumschicht dient als Ätzstopp bei der partiellen Entfernung der PECVD-Oxid Opferschicht zur Erzeugung der Kavität. Die obere Funktionsschicht besteht wiederum aus amorphen Silizium. Eine Schädigung des Schichtenstapels während der isotropen Oxidentfernung zur Kavitätserzeugung tritt bei Verwendung der angegebenen Parameter nicht auf.

Eine erste Untersuchung der elektrischen Eigenschaften der amorphen Siliziumschicht erfolgt durch die Verwendung einer Teststruktur. Dabei wird eine rund $90 \mathrm{~nm}$ Dicke Siliziumdioxid-Schicht erzeugt, auf der die amorphe Siliziumschicht abgeschieden wird. Das Aluminium als Metallisierung wird mittel thermischen Aufdampfens aufgebracht und im Lift-Off Verfahren entsprechend der in Abbildung 5 gezeigten Maske des späteren Sensors strukturiert. Abbildung 8 zeigt das Ergebnis der elektrischen Vermessung der Struktur. Deutlich zu erkennen ist sowohl der durch positive als auch durch negative Ladungsträger hervorgerufene Drainstrom, welcher sich im ambipolare Schaltverhalten des Bauteils darstellt.

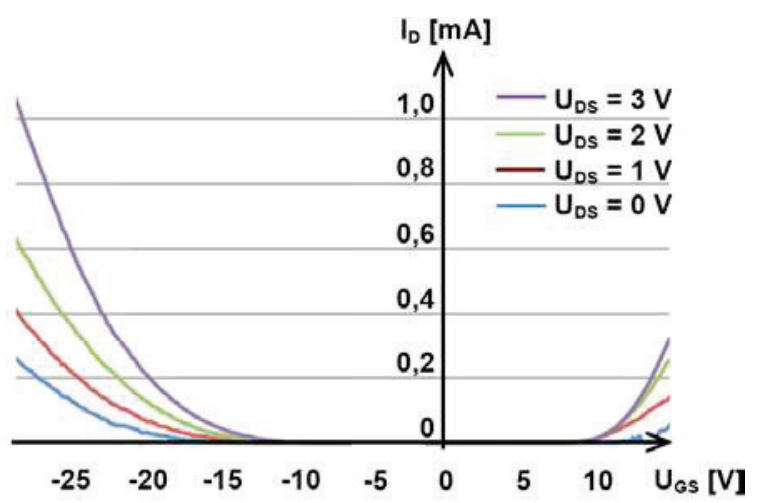

Abb.. 8: Transferkennlinienfeld eines auf amorphen Silizium gefertigten $\psi$ MOS-Feldeffekttransistors mit einer Kanallänge von $4 \mu m$ und -weite von $50 \mu \mathrm{m}$.

Abschließend erfolgt die Analyse eines $\psi$ MOS-Feldeffekttransistors mit Gate aus Titannitrid.

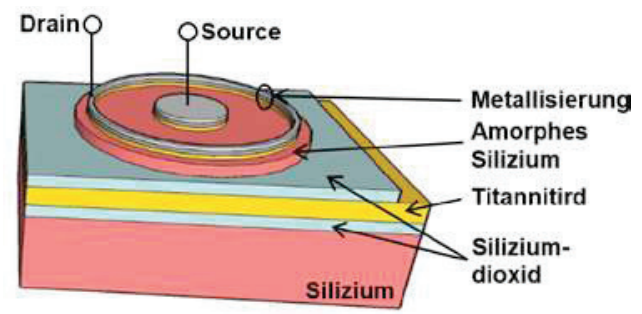

Abb.. 9: Schematische Darstellung eines $\psi$ MOS-FETS aus amorphen Silizium mit einer Metallelektrode als Gateanschluss. 
Hierbei wird entsprechend der in Abbildung 9 gezeigten schematischen Struktur das Gate unmittelbar unterhalb des Oxides kontaktiert, wodurch sich die Gatespannung nicht mehr durch den als parasitären Widerstand wirkenden Siliziumwafer erst aufbauen muss. Dieses Verfahren verspricht eine bessere Kontrolle über das Gate und geringere Spannungen. Gleichzeitig erfolgt zur Vermeidung von Oberflächenschäden infolge einer trockenchemischen Ätzung die Strukturierung des amorphen Siliziums nasschemisch mittels einer Säuremischung aus:

- $100 \mathrm{ml}$ Salpetersäure $\left(\mathrm{HNO}_{3}\right)$

- $5 \mathrm{ml}$ gepufferte Flusssäure $\left(\mathrm{HF}+\mathrm{NH}_{4} \mathrm{~F}\right)$

- $100 \mathrm{ml}$ Wasser $\left(\mathrm{H}_{2} \mathrm{O}\right)$.

Dabei wird das Silizium durch die Salpetersäure zunächst oxidiert, gefolgt von einem Abtrag des entstandenen Oxides durch die Flusssäure. Dieser Prozessablauf führt zu den gewünschten Strukturen, wobei die Unterätzungen aufgrund der gewählten Strukturabmessungen unkritisch sind. Jedoch offenbart die elektrische Vermessung der so hergestellten Bauelemente erhebliche Nachteile dieses Verfahrens. Wie Abbildung $10 \mathrm{zu}$ entnehmen ist, findet ein Ladungsträgertransport, hier gezeigt für Elektronen, zwar statt, resultiert jedoch in einem wesentlich geringeren Drainstrom.

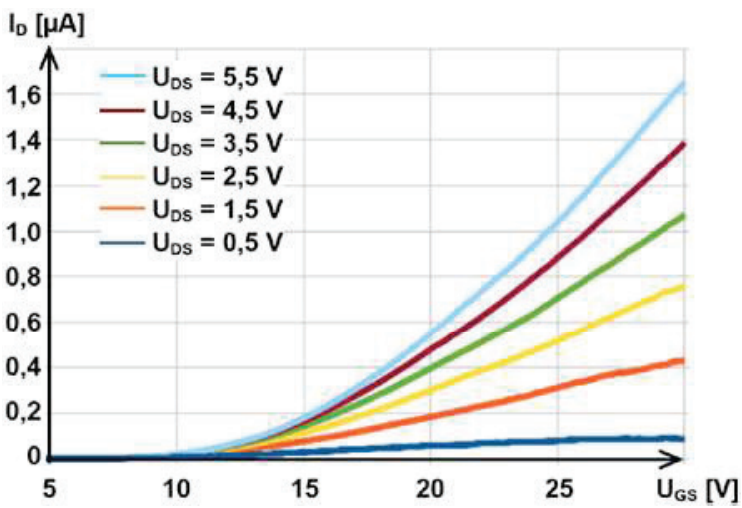

Abb.. 10: Transferkennlinienfeld eines auf amorphen Silizium gefertigten $\psi$ MOS-Feldeffekttransistors mit runder Kanalform.

Die Ursache hierfür ist in der Verwendung der Ätzlösung begründet. Die oxidierende Wirkung der Salpetersäure läuft demnach nicht nur an der Oberfläche ab. Eine Diffusion in die amorphe Schicht aus Silizium senkt die halbleitenden Eigenschaften des Werkstoffes herab, wodurch der Drainstrom sinkt. Da beim SOISubstrat die Top-Silizium Schicht monokristallin ist, tritt dieser Effekt bei der Verwendung dieses Substrates nicht auf. Aus diesem Grund ist in weiteren Untersuchungen das amorphe Silizium zwingend trockenchemisch zu struktu- rieren, um die elektrischen Eigenschaften nicht negativ zu beeinflussen.

\section{Zusammenfassung und Ausblick}

Die Nutzung eines Transistors zur Wandlung der mechanischen Verspannung einer Siliziummembran in ein elektrisches Signal ist anhand der druckabhängigen Kennlinienverschiebung am Beispiel eines $\psi$-MOS-FETs gezeigt worden. Eine konzeptionelle Erweiterung zur Steigerung der Sensitivität und zur Senkung des Stromverbrauches ist ebenfalls aufgeführt. Des Weiteren ist die Eignung von amorphen Silizium aus einer Niedertemperaturabscheidung als Grundlage für die Realisierung von Sensoren auf einer vorprozessierten Schaltung untersucht worden.

In folgenden Untersuchungen wird ein vollständiger Drucksensor auf Basis dieses Werkstoffes hergestellt und mit der Realisierung auf SOI Substrat verglichen. Der Vergleich dieser Wandungsart mit der konventionellen resistiven Ausführung steht ebenfalls noch aus. Zudem wird der Ansatz zur Wandlung der Verspannung einen nanoskaliken Transistor zu verwenden weiter verfolgt.

\section{Literaturverzeichnis}

[1] B. Bhushan, Springer Handbook of Nanotechnology, 1756-1757 (2006)

[2] G. Zimmer, K. Trieu, Forum Forschung 2004/2005, 96-100 (2004)

[3] A. J. Shar, B. A. Davis, M. J. Collins, L. G. Carney, IEEE Transactions on Biomedical Engineering 56 (10), 2512-2517 (2009)

[4] Vgl.: H. Takahashi, K. Matsumoto, I. Shimoyama, Conference Publications on 22th IEEE International Conference on Micro Electro Mechanical Systems, S. 825-828 (2009)

[5] L- He, S. Elassaad, Y. Shi, System-in-Package: Electrical and Layout Perspectives" Now Publishers Inc. (2011)

[6] L.-T. Wang, Ch. E. Stroud, N. A. Touba, System-on-Chip Test Architectures: Nanometer Design for Testability, Elsevier (2008)

[7] A. Witvrouw, F. Van Steenkiste, D. Maes, L. Haspeslagh, P. Van Gerwen, P. De Moor, S. Sedky, C. Van Hoof, A. C. de Vries, A. Verbist, A. De Caussemaeker, B. Parmentier, K. Baert, Microsystems Technologies 6 (5), 192-199 (2000)

[8] E. Hering, G. Schönfelder, Sensoren in Wissenschaft und Technik - Funktionsweise und Einsatzgebiete, 6-12, Vieweg + Teubner Verlag (2012)

[9] O. Brand, G.K. Fedder, Advanced Micro \& Nanosystems, Volume 2 - CMOS-MEMS, 303f., Wiley-Vch Verlag GmbH \& Co. KGaA (2004)

[10] Z. Zhang, Y.-H. Zhang, L.-T. Liu, T.-L. Ren, Proceedings of the IEEE Sensors 2008 Conference, 1564-1567, (2008)

[11] S. E. Thompson, M. Armstrong et al., IEEE Electron Device Letters 25 (4), 191-193 (2004)

[12] M. V. Fischetti, Journal of Applied Physics, 92 (12), 7320-7324 (2002) 
[13] J. Knoch, K. Kallis, Mikromechanischer Drucksensor und Verfahren zu dessen Herstellung 'SCHON-FET', Patentanmeldung WO 2012/095117 A1 (2012)

[14] S. Renard, Journal of Micromechanics and Microengineering 10, 245-249 (2000)

[15] S. Cristoloveanu, IEEE Transactions on Electron Devices 47 (5) (2000)

[16] U. Hilleringmann, Silizium Halbleitertechnologie, S. 138-144, B. G. Teubner (2002) 